\title{
APLICABILIDADE DA SIMULAÇÃO TÉCNICO-ECONÔMICO NA BOVINOCULTURA DE CORTE DE MATO GROSSO DO SUL
}

\author{
APPLICABILITY OF TECHNICAL-ECONOMICAL SIMULATION IN BEEF CATTLE \\ PRODUCTION IN MATO GROSSO DO SUL, BRAZIL

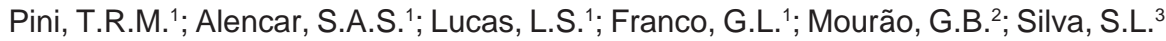 \\ e Brumatti, R.C. ${ }^{1 *}$
}

\begin{abstract}
${ }^{1}$ Faculdade de Medicina Veterinária e Zootecnia. Universidade Federal de Mato Grosso do Sul. Campo Grande, MS. Brasil. *ricardo.brumatti@ufms.br

${ }^{2}$ Escola Superior de Agricultura Luiz de Queiroz. Universidade de São Paulo. Piracicaba, SP. Brasil.

${ }^{3}$ Faculdade de Zootecnia e Engenharia de Alimentos. Universidade de São Paulo. Pirassununga, SP. Brasil.
\end{abstract}

\section{PalaVRas ChaVE ADICIONAIS}

Modelagem. Simulador pecuário. Sistema computacional.

\section{RESUMO}

Como ferramentas auxiliares na tomada de decisões para o produtor rural, programas computacionais de simulação têm sido estudados e desenvolvidos por vários pesquisadores. Nesta linha de estudo, testes com o sistema de simulação técnico-econômico foram realizados, sendo aplicados junto a produtores rurais e com os índices da bovinocultura de corte brasileira. Os testes com produtores rurais geraram resultados com padrões econômicos próximos dos alcançados na prática. Quanto aos cenários nacionais, é interessante observar que o lucro com com o kilo produzido se manteve relativamente constante, com um acréscimo de menos de $\mathrm{R} \$ 0,20$, da média brasileira para o sistema melhorado 2, ou seja, $6,91 \%$, porém, quando se observa a EBIT, constata-se um aumento de $185,14 \%$. Isto, se deve ao aumento da quantidade total de kilos produzidos entre um cenário e outro, que aumentou de $125 \mathrm{t}$ para $310 \mathrm{t}$, devido principalmente aos incrementos com nutrição e ao encurtamento do ciclo produtivo em decorrência dos melhores índices zootécnicos e consequentemente ao incremento no lucro em $\mathrm{R} \$$ /ha/ano. Os resultados obtidos da simulação com os índices da bovinocultura de corte nacional foram condizentes com os índices produtivos

\#Trabalho realizado com apoio do CNPq, FUNDECT/MS e UFMS. Cotação do dólar: $\mathrm{US} \$ 1.00=\mathrm{R} \$ 2,03$.

\author{
AdDiTIONAL KEYWORDS \\ Computer system. Livestock simulation. Modeling.
}

apresentados, mostrando uma concordância entre ambos, o que demonstra a eficácia de se utilizar o sistema de simulação técnico-econômico na análise de fazendas produtoras de bovinos de corte.

\section{SUMMARY}

As auxiliary tools in decision making for farmers, computer simulation programs have been studied and developed by several researchers. In this line of study, tests with technical economic simulation system were performed, applied together with farmers and with the indices of Brazilian beef cattle. The results of tests applied with farmers showed similar results to those achieved by the market. As regards national scenarios, it is interesting to note that the profit kilo produced is kept relatively constant, with an increase of less than $\mathrm{R} \$ 0.20$, the national average for the improved system 2 , ie $6.91 \%$, but when you look at the EBIT, there is an increase of $185.14 \%$. This was due to increased total amount produced between one scene and another, which increased from 125 to $310 \mathrm{t}$, mainly due to increases in nutrition and shortening the production cycle as a result of the best indexes and hence the increase in profit in $\mathrm{R} \$$ / ha/year. The results of simulation with the contents of the national beef cattle production were consistent with the contents presented, showing 
a correlation between both, which demonstrates the effectiveness of using the technical economic simulation system for analysis farms that producing beef cattle.

\section{INTRODUÇÃO}

Devido a competitividade, complexidade e dinâmica crescente no setor pecuário brasileiro, e como não há fórmulas ou recomendações únicas a serem empregadas na bovinocultura de corte, é necessária a busca constante de novas visões administrativas e gerenciais pelo produtor rural (Fontoura-Júnior et al., 2007; Carvalho et al., 2008).

Neste contexto é fundamental o aprimoramento e disseminação de tecnologias em diferentes áreas como genética, manejo, nutrição e sanidade que são responsáveis pelo incremento da produtividade na bovinocultura de corte. Com essa premissa, diversas pesquisas propuseram o uso de programas computacionais de simulação para aferir os resultados econômicos dos sistemas produtivos e analisar sua sustentabilidade (Guimarães et al., 2005; Costa et al., 2006; Fontoura-Júnior et al., 2007; Brumatti et al., 2011).

Os modelos de simulação oferecem suporte nas áreas de nutrição, melhoramento genético, reprodução e sanidade, onde visa atingir uma simplificação da realidade (Braga et al., 1997).

A máxima eficiência biológica não coin-
Tabela I. Índices zootécnicos médios do rebanho brasileiro e em sistemas envolvendo cria, recria e engorda com uso mais intensivo de tecnologia. (Average performance indexes of the Brazilian herd and life-cycle beef cattle production systems with more intensive use of technology).

\begin{tabular}{lccc}
\hline & MB & \multicolumn{2}{c}{ Sistemas } \\
melhorados \\
Índices & & 1 & 2 \\
\hline Natalidade (\%) & 60 & $>70$ & $>80$ \\
Mortalidade até a desmama (\%) & 8 & 6 & 4 \\
Taxa de desmama (\%) & 55 & $>66$ & $>77$ \\
Mortalidade pós-desmama (\%) & 4 & 3 & 2 \\
Idade à primeira cria (anos) & 4 & 3 & 2 \\
Intervalo entre partos (meses) & 20 & $<17$ & $<15$ \\
Idade média de abate (anos) & 4 & 3 & 2 \\
Taxa de abate (\%) & 17 & 20 & 35 \\
Peso médio de carcaça (kg) & 210 & 230 & 240 \\
Rendimento de carcaça (\%) & 53 & 54 & 57 \\
Taxa de lotação (animal/ha) & 0,9 & 1,2 & 1,6 \\
Peso de carcaça kg/ha & 34 & 53 & 80 \\
& & & \\
\hline
\end{tabular}

$\mathrm{MB}=$ Média brasileira.

Fonte: Modificado de Zimmer e Euclides Filho (1997), citado por Euclides Filho (2000).

cide sempre com a melhor eficiência e lucratividade econômica, portanto, é fundamental que os modelos de simulação considerem os principais fatores envolvidos na produção de bovinos de corte, para que os resultados obtidos sejam próximos da

Tabela II. Características gerais, obtidas por questionário, de 154 propriedades rurais de Mato Grosso do Sul. (General characteristics, obtained by questionnaire from 154 farms in Mato Grosso do Sul).

\begin{tabular}{|c|c|c|c|c|c|}
\hline & Média & Moda & Mediana & Quantidade & $\%$ \\
\hline Tempo da propriedade (anos) & 23,62 & 10 & 20 & - & - \\
\hline Área (ha) & 1159,11 & 1500 & 465 & - & - \\
\hline \multicolumn{6}{|l|}{ Tipo de produção } \\
\hline Cria & - & & - & 45 & 29,22 \\
\hline Engorda & - & & - & 65 & 42,21 \\
\hline Ciclo completo & - & & - & 44 & 28,57 \\
\hline
\end{tabular}

Archivos de zootecnia vol. 63, núm. 241, p. 192. 
realidade bioeconômica da atividade (Fontoura-Júnior et al., 2007).

Este estudo teve por objetivo avaliar um sistema de simulação técnico-econômico de cenários produtivos da bovinocultura de corte.

\section{MATERIALE MÉTODOS}

Para o desenvolvimento do trabalho foi utilizado parte da metodologia do sistema técnico-econômico apresentado por Brumatti et al.(2011).

Tabela III. Avaliação econômica dos cenários produtivos agrupados de acordo com o sistema produtivo, obtidas através da aplicação de questionários à produtores rurais. Valores monetários em $R \$$. Área em hectare. (Economic evaluation of the production scenarios grouped according to the production system, obtained through the application of questionnaires to farmers. Monetary values in $\mathrm{R} \$$. Area in hectare).

\begin{tabular}{|c|c|c|c|}
\hline & Cria & Engorda & $\mathrm{CC}$ \\
\hline Área & 1094,6 & 728,2 & 1861,7 \\
\hline - de pastagem & 875,7 & 582,5 & 1489,3 \\
\hline Valor da terra ${ }^{1}$ & 2742,5 & 2572,1 & 2663,1 \\
\hline Receita bruta & 201208,9 & 692100,0 & 34717,2 \\
\hline \multicolumn{4}{|l|}{ Custos } \\
\hline Insumos $^{2}$ & 75335,6 & 523963,7 & 78102,0 \\
\hline Mão de obra & 44054,6 & 42976,4 & 73727,8 \\
\hline Total & 119390,2 & 566940,1 & 51829,8 \\
\hline \multicolumn{4}{|l|}{ Despesas } \\
\hline Administrativas & $s^{3} 4211,5$ & 1617,1 & 6248,3 \\
\hline Imposto 4 & 6837,7 & 3888,9 & 10846,1 \\
\hline Total & 11049,1 & 5506,0 & 17094,4 \\
\hline EBITDA & 70769,6 & $119.65,88$ & 265793,0 \\
\hline EBITDA/hectare & e 64,65 & 164,32 & 142,77 \\
\hline \multicolumn{4}{|c|}{$\begin{array}{l}\mathrm{CC}=\text { Ciclo completo; EBITDA= lucros antes de } \\
\text { juros, impostos, depreciação e amortização. }{ }^{1} \mathrm{R} \$ / \\
\text { ha; }{ }^{2} \text { Insumos e reprodução. }{ }^{3} \text { Administração, } \\
\text { consultoria e diversos. }{ }^{4} \text { Imposto sobre a } \\
\text { Propriedade territorial rural. }\end{array}$} \\
\hline
\end{tabular}

O simulador foi desenvolvido com suporte computacional de um microcomputador e planilhas eletrônicas do Excel 2007®, pacote Microsoft Office 2007®, sendo que este se trata de um sistema determinístico e simula rebanhos nas atividades de cria; recria; ciclo completo; ciclo completo com seleção de tourinhos; e engorda, tanto para um número fixo de matrizes, quanto para um número fixo de animais para engorda, integrando custos e receitas anuais dos diversos cenários simulados.

Este se norteia pela interação de três grandes centros de cálculos, sendo eles: o simulador de rebanho; os índices zootécnicos; e os centros de controle de atividades de custos e receitas.

Para suprir o simulador com informações foi realizada uma coleta de dados nos meses de outubro, novembro e dezembro 2011, por meio da aplicação de 154 questionários e quatro entrevistas diretas à produtores rurais de municípios de Mato Grosso do Sul.

Os questionários foram elaborados de maneira a contemplar as características físicas da fazenda e os principais índices zootécnicos, além das condições socioculturais do proprietário e dos empregados. Com ele se objetivou rapidez e praticidade nas coletas de dados.

Nas entrevistas foram abordadas questões acerca das características da fazenda, dos índices zootécnicos e dos manejos sanitários, reprodutivos, de pastagens e gerenciais.

Os dados socioculturais coletados foram: sexo do entrevistado, idade, faixa etária, nível de escolaridade. Para se caracterizar as propriedades foram coletados: localização da fazenda, tempo de exploração, área total em hectares (ha), quantidade de funcionários e nível educacional médio da mão-de-obra.

Os índices zootécnicos coletados foram: taxa de lotação, taxas de descartes, taxas de mortalidades, taxas de fertilidades, todas para as diversas categorias animais que as 
compõem, além das atividades realizadas junto ao calendário sanitário, plano nutricional, plano reprodutivo em termos de monta natural e uso de inseminação artificial, e plano anual de reforma e manutenção de pastagens. Uma vez inseridos tais dados, o ganho de peso médio para cada categoria foi estimado para a obtenção dos animais para abate.

O valor da terra, em hectares, foi determinado através do município em que a fazenda estava localizada, perfil do solo e da capacidade de suporte da pastagem, sendo caracterizados em três tipos: cerrado; pastagem formada de baixo suporte; e pastagem formada de alto suporte (Anualpec, 2010).

A comparação foi realizada com cenários simulados através dos índices produtivos e zootécnicos nacionais citados por Euclides Filho (2000) apresentados na tabela I. Foram simulados três cenários, denominados: Média brasileira; Melhorado 1; e Melhorado 2. Demais características de manejo, assim como índices produtivos e zootécnicos não citados foram determinados de acordo com os padrões observados na bovinocultura de corte de Mato Grosso do Sul, sendo que a área da propriedade rural foi padronizada em 3500 hectares para os três cenários e foi atribuído o valor do hectare do município de Campo Grande com pastagem formada de alto suporte (Anualpec, 2010).

As simulações dos cenários Melhorado 1 e 2 foram realizadas através do incremento de atividades ao cenário Média brasileira, dentro de limites realísticos que justificassem seus desempenhos superiores. Portanto, para o cenário Melhorado 1, foi atribuído: incrementos nutricionais com pastagem formada de alto suporte, sal mineral adicionado de ureia para matrizes e bois de 24 e 36 meses de idade durante três meses, e proteico para seca durante dois meses para as duas últimas categorias; aquisição de implementos agrícolas; benfeitorias em cerca e casa para funcionário, e contratação de um peão. No
Melhorado 2, somado ao já citado anteriormente, foi adicionado: incrementos nutricionais como, sal mineral adicionado de ureia e proteico para seca aos garrotes e touros durante três meses e dois meses, respectivamente, e fornecimento de proteico para seca durante quatro meses para bois de 24 meses de idade; aquisição de trator e implementos agrícolas; aumento em 5 \% e 10 \% nas áreas de reforma e manutenção de pastagens, respectivamente, além de incrementos em suas atividades; contratação de tratorista e assessoria zootécnica.

Para elaboração dos Demonstrativos de Resultados Econômicos (DRE) foram utilizados valores correntes médios de mercado de insumos e de animais, cotados entre fevereiro e março de 2011, em Campo Grande, MS.

O Imposto sobre a Propriedade Territorial Rural (ITR) foi descontado antes da EBITDA (earnings before interest, taxes, depreciation, and amortization, que em português significa lucros antes de juros, impostos, depreciação e amortização), porque diferentemente de outros impostos, este não é calculado sobre receitas ou lucros. Sua elaboração foi realizada de acordo com a legislação vigente e, para tanto, foi padronizado o grau de utilização da terra em $80 \%$ e o valor da terra nua foi estimado através do Anualpec (2010), onde para os municípios que não eram citados neste, foi adotado o valor do município mais próximo geograficamente.

\section{RESULTADOSEDISCUSSÃO}

Na tabela II são apresentadas as características das 154 propriedades rurais.

Na tabela III visualizam-se os DRE médios, por sistema de produção, das 154 propriedades rurais.

Segundo Simões et al. (2006), na avaliação econômica comparativa dos sistemas de produção no município de Aquidauana, Mato Grosso do Sul, a atividade de engorda apresentou Margem 
Bruta/ha, que é equivalente à EBITDA/ha, maior que a de cria. Os resultados encontrados neste estudo corroboram a maior relação EBITDA/ha do sistema de produção de engorda, seguida de ciclo completo e cria encontrado por Simões et al. (2006).

Dentre os sistemas de produção o que apresentou a maior EBITDA/ha por hectare foi o de engorda, apesar do valor despendido com a aquisição de animais de reposição representar $71 \%$ dos gastos. Este valor é próximo dos 67,46 \% descrito por Simões et al.(2006).
Os mesmos autores observaram que o sistema de produção de cria possuiu uma distribuição de gastos mais equitativa do que os demais sistemas, o que também foi constatado neste estudo.

Nos três tipos de produção a EBITDA cobriu o gastos, o que sugere que em média, as atividades desenvolvidas pelas 154 fazendas são sustentáveis em curto prazo. Devido a diversidade de áreas observadas entre estas fazendas, não foi possível obter no questionário informações acerca das atividades de reforma e manutenção de

Tabela IV. Avaliação econômica e financeira de quatro propriedades através do sistema de simulação técnico-econômico. Valores monetários em R\$. Área em hectare. (Economic and financial evaluation of four properties through the techno-economic simulation system. Monetary values in $\mathrm{R} \$$. Area in hectare).

\begin{tabular}{|c|c|c|c|c|}
\hline & $\begin{array}{c}\text { Fazenda } 1 \\
\text { Cria }\end{array}$ & $\begin{array}{c}\text { Fazenda } 2 \\
\text { Engorda }\end{array}$ & $\begin{array}{c}\text { Fazenda } 3 \\
\text { Ciclo completo }\end{array}$ & $\begin{array}{c}\text { Fazenda } 4 \\
\text { Ciclo completo } \\
\text { (Rebanho de elite) }\end{array}$ \\
\hline Área & 1280,0 & 630,0 & 368,0 & 299,0 \\
\hline - de pastagem & 1024,0 & 504,0 & 294,4 & 239,2 \\
\hline Valor da terra $\mathrm{R} \$ / \mathrm{ha}$ & 3500,0 & 1683,0 & 2000,0 & 3500,0 \\
\hline Receita bruta & 225541,3 & 295219,5 & 90434,6 & 375240,9 \\
\hline \multicolumn{5}{|l|}{ Custos } \\
\hline Insumos $^{1}$ & 111444,4 & 232 118,4 & 31599,5 & 61544,9 \\
\hline Mão de obra & 42405,5 & 15902,1 & 15902,1 & 31804,2 \\
\hline Manutenção & 56858,5 & 21877,4 & 23018,0 & 40701,0 \\
\hline Total & 210708,4 & 269897,9 & 70519,6 & 134050,0 \\
\hline \multicolumn{5}{|l|}{ Despesas } \\
\hline Administrativas $^{2}$ & 17696,8 & 16741,3 & 14311,2 & 22798,2 \\
\hline Impostos ${ }^{3}$ & 13440,0 & 736,0 & 1590,4 & 1046,5 \\
\hline Total & 31 136,8 & 17477,27 & 15901,6 & 23844,7 \\
\hline EBITDA & $-16303,9$ & 7844,4 & 4013,4 & 217346,2 \\
\hline Depreciações & 48684,0 & 18620,3 & 15677,3 & 24686,3 \\
\hline EBIT & $-64987,9$ & $-10775,9$ & $-11663,9$ & 192659,9 \\
\hline EBITDA/ha & $-12,74$ & 12,45 & 10,91 & 726,91 \\
\hline EBIT/ha & $-50,77$ & $-17,11$ & $-31,70$ & 644,35 \\
\hline
\end{tabular}

${ }^{1}$ Insumos, pastagem e reprodução; ${ }^{2} \mathrm{Adm}$ inistração, consultoria e diversos; ${ }^{3}$ Imposto sobre a propriedade territorial rural.

EBITDA= lucros antes de juros, impostos, depreciação e amortização; EBIT= lucros antes de juros, impostos. 
Tabela V. Análise econômica de três cenários produtivos através do sistema de simulação técnico-econômico, utilizando índices zootécnicos apresentados por Euclides Filho, 2000. Valores monetários em $\mathrm{R} \$$. Área em hectare. (Economic analysis of three scenarios production through the techno-economic simulation system, using biological indices presented by Euclides Filho 2000. Monetary values in R\$. Area in hectare).

\begin{tabular}{|c|c|c|c|}
\hline \multirow[b]{2}{*}{ Índices } & \multirow[t]{2}{*}{ MB } & \multicolumn{2}{|c|}{$\begin{array}{l}\text { Sistemas } \\
\text { melhorados }\end{array}$} \\
\hline & & 1 & 2 \\
\hline Área & 3.500 & 3.500 & 3.500 \\
\hline - de pastagem & 2.800 & 2.800 & 2.800 \\
\hline Receita bruta & 743948 & 119207 & 1848120 \\
\hline \multicolumn{4}{|l|}{ Custos } \\
\hline Insumos $^{1}$ & 207346 & 348259 & 544263 \\
\hline Mão-de-obra & 58308 & 74210 & 90112 \\
\hline Manutenção & 59424 & 65303 & 102.885 \\
\hline Total & 325078 & 487772 & 737.260 \\
\hline \multicolumn{4}{|l|}{ Despesas } \\
\hline Administrativa $^{2}$ & 20771 & 21988 & 48700 \\
\hline Impostos 3 & 36750 & 36750 & 36750 \\
\hline Total & 57521 & 58738 & 85450 \\
\hline EBITDA & 361349 & 572697 & 1.025 .410 \\
\hline Depreciações & 20.245 & 51229 & 52769 \\
\hline EBIT & 341.104 & 521468 & 972.641 \\
\hline carcaça, kg/ha & 35,7 & 53,8 & 88,60 \\
\hline Lucro/UA/ano & 149,0 & 170,0 & 225,0 \\
\hline Lucro/kg/ano & 1,53 & 1,59 & 1,74 \\
\hline Lucro/ha/ano & 133 & 204 & 359 \\
\hline
\end{tabular}

$\mathrm{MB}=$ Média brasileira.

${ }^{1}$ Insumos, pastagem e reprodução; ${ }^{2} \mathrm{Administração,}$ consultoria e diversos; ${ }^{3}$ Imposto sobre a propriedade territorial rural.

EBITDA= lucros antes de juros, impostos, depreciação e amortização; EBIT= lucros antes de juros, impostos.

pastagens, benfeitorias e equipamentos agrários. Com isso, não se pode calcular os gastos com manutenção e depreciação, o que forneceria base para análise da sustentabilidade em longo prazo.

As análises econômicas e financeiras das quatro propriedades rurais estão evidenciadas na tabela IV.

Em todas as propriedades, com exceção a Propriedade 1, verificou-se EBITDA positiva, ainda assim, os valores da EBITDA/ha (Margem bruta/ha) foram distantes dos apresentados por Simões et al. (2006), que para atividade de cria encontraram R $\$ 175,61$ e de engorda de R\$935,81. Costa et al. (2006), analisando um sistema de ciclo completo com manejo tradicional, no Rio Grande do Sul, encontraram EBITDA/ha (Margem bruta/ha) de $\mathrm{R} \$ 87,39$, enquanto no presente estudo foi de R\$10,91. A Propriedade 4, com sistema de ciclo completo com rebanho de elite, obteve a maior EBITDA/ha, R \$726,91, sendo 66,63 vezes superior a de ciclo completo com rebanho comercial. Quando avaliada a EBIT, somente a esta foi positiva, resultando em uma EBIT/ha de R\$644,35.

O item que mais onerou os custos foram os insumos, seguidos de manutenções e depreciações. A aquisição de animais para reposição teve maior impacto na atividade de engorda, onde representou 56,67 \% dos gastos.

O valor despendido na aquisição de animais de reposição explica a distribuição de gastos mais equitativa do sistema de ciclo completo em relação ao de recria/engorda (Simões et al. 2006; Tavares et al. 2009). Em uma análise da rentabilidade econômica da bovinocultura de corte de nove regiões do estado de Goiás, Tavares et al. (2009) constataram que os itens que mais oneraram a atividade foram: aquisição de animais; suplementação animal; mão de obra; e manutenção de pastagens.

A propriedade que apresentou maior equidade na distribuição dos componentes de gastos foi a de ciclo completo com rebanho de elite, seguida da de ciclo completo com rebanho comum. Este fato discorda do encontrado na análise realizada com dados 
Tabela VI. Análise produtiva-econômica com base no sistema de simulação técnicoeconômico de duas fazendas de ciclo completo. Valores monetários em $R \$$. (Productiveeconomic analysis based on the techno-economic simulation of two life-cycle beef cattle production systems. Monetary values in $\mathrm{R} \$$. Area in hectare).

\begin{tabular}{lcccc}
\hline & $\begin{array}{c}\text { Carcaça } \\
\text { kg/ha }\end{array}$ & UA/ano & $\begin{array}{l}\text { Lucro } \\
\text { kg/ano }\end{array}$ & ha/ano \\
\hline Fazenda 3/Ciclo completo & 40,60 & $-16,00$ & $-0,14$ & $-14,00$ \\
Fazenda 4/Ciclo completo $^{1}$ & 88,90 & 516,00 & 6,05 & 841,00 \\
\hline
\end{tabular}

UA= Unidade animal (450 $\mathrm{kg}$ peso vivo). ${ }^{1}$ Rebanho de elite.

obtidos por questionários e com o apresentado por Simões et al. (2006), onde ambos demonstraram essa característica para o sistema de produção de cria.

Os índices produtivos e econômicos dos cenários nacionais Média brasileira; Melhorado 1; e Melhorado 2 podem ser visualizados na tabela $\mathbf{V}$.

Dentre os índices produtivos, Euclides Filho (2000) estimou para os três cenários a quantidade de quilogramas de carcaça produzidas por hectare, sendo respectivamente, para a Média brasileira, Sistema melhorado 1 e 2 de: 34; 53; e 80 kg carcaça/ ha, respectivamente. Ao analisar os dados obtidos das simulações, observa-se que os valores são próximos, principalmente para os dois primeiros cenários.

Vale mencionar que no estudo supra citado, dos 12 índices apresentados pelo autor, utilizaram-se nove para gerar os cenários produtivos. Isto não ausenta a necessidade de fornecer a maior quantidade possível de dados para o simulador a fim de obter resultados que reflitam a realidade da fazenda, porém se observa que com o emprego dos principais índices zootécnicos, como taxa de lotação, natalidade, mortalidades por categoria, idade à primeira cobertura e cria, idade média de abate, peso médio e rendimento de carcaça, e com o fornecimento de dados básicos das características da propriedade, se alcança resultados econômicos satisfatórios.
Analisando a produção de carcaça por hectare e o lucro discriminado das Fazendas 3 e 4 (tabela VI) observa-se que apesar da Fazenda 3 produzir maior quantidade que a média brasileira, possui lucro negativo e isso está relacionado a altos gastos, em contraste com a Fazenda 4 que apesar de produzir 88,90 Kg de carcaça/ha, que é 11,13 $\%$ superior ao Sistema melhorado 2, possui um lucro, ha/ano, 134,26 \% maior. Isto está atrelado ao valor agregado de seu principal produto de comercialização, o tourinho.

É interessante observar que o lucro o kilo produzido nos três cenários obtidos sob os índices apresentados por Euclides Filho (2000), se mantém relativamente constante, com um acréscimo de menos de R\$ 3,00, da média brasileira para o Sistema melhorado 2, ou seja, 6,91 \%, e no entanto, quando se observa a EBIT, constata-se um aumento de $185,14 \%$. Isto, como evidenciado pelas simulações, deveu-se ao aumento da quantidade total de kilos produzidos entre um cenário e outro, que aumentou de 125 para 310 t, devido principalmente aos incrementos com nutrição e ao encurtamento do ciclo produtivo em decorrência dos melhores índices zootécnicos e consequentemente ao incremento no lucro em $\mathrm{R} \$$ / ha/ano.

Esta característica é levantada por Somwaru e Valdes (2004), que salientaram o aumento da eficiência nas atividades acerca da produção nas propriedades com maior 
área territorial, devido à redução de custos relativos. A razão é que os custos fixos e de oportunidade, que compreende a propriedade rural, implementos e benfeitorias, permanecem constantes, ao passo que o custo unitário total tende a diminuir com o aumento na produtividade e no número de animais do rebanho (Barbosa et al., 2010).

\section{BIBLIOGRAFIA}

Anualpec. Anuário da Pecuária Brasileira. 2010. IFNP. São Paulo, SP. Brasil.

Barbosa, F.A.; Graça, D.S.; Andrade, V.J.; Cezar, I.M.; Santos, G.G. e Souza, R.C. 2010. Produtividade e eficiência econômica de sistemas de produção de cria, recria e engorda de bovinos de corte na região sul do estado da Bahia. Arq Bras Med Vet Zootec, 62: 677685.

Braga, R.; Pinto, P. e Coelho, J. 1997. Os modelos de simulação e a investigação de sistemas da agricultura. Ingenium, 20: 48-54.

Brumatti, R.C.; Ferraz, J.B.S.; Eler, J.P. e Formigoni, I.B. 2011. Desenvolvimento de índice de seleção em gado corte sob o enfoque de um modelo bioeconômico. Arch Zootec, 60: 205-213.

Carvalho, T.B.; De Zen, S. e Ferreira, P.C. 2008. Caracterização da atividade pecuária de engorda nos principais países produtores de carne bovina. Congresso da Sociedade Brasileira de Economia, Administração e Sociologia Rural. Anais... Rio Branco.

Costa, L.B.; Ceretta, P.S. e Gonçalves, M.B.F. 2006. Viabilidade econômica: análise da bovinocultura de corte. Inf Econ, 26: 26-38.

Euclides Filho, K. 2000. Produção de bovinos de corte e o trinômio genótipo-ambiente-mercado. Documentos/Embrapa Gado de Corte. Campo Grande, MS. 85. 61 pp.

Fontoura-Júnior, J.A.S.; Menezes, L.M.; Corrêa, M.N. e Dionello, N.J.L. 2007. Utilização de mode-

\section{CONCLUSÕES}

A partir da análise dos dados, os resultados econômicos apresentados pelo sistema de simulação técnico-econômico foram condizentes com a realidade do mercado, demonstrando a eficácia de utilizá-lo na análise de fazendas produtoras de bovinos de corte e elite.

los de simulação em sistemas de produção de bovinos de corte. Vet Zootec, 14: 19-30.

Guimarães, P.H.S.; Madalena, F.E. e Cezar, I.M. 2005. Simulação dos efeitos dos preços de produtos e insumos na avaliação econômica de três sistemas alternativos de bovinocultura de cria. Arq Bras Med Vet Zootec, 57 (supl. 2): 227-230.

Simões, A.R.; Gama, F.F.C.; Canuto, N.G.D. e Carvalho, D.M.G. 2006. Avaliação econômica comparativa de sistemas de produção de gado de corte na região de Aquidauana-MS. Congresso da Sociedade Brasileira de Economia, Administração e Sociologia Rural, 44. Anais... Fortaleza.

Somwaru, A. and Valdes, C. 2004. Brazil's beef production and its efficiency: A comparative study of scale economies. GTAP $7^{\text {th }}$ Annual Conference on Global Economic Analysis. https:/ /www.gtap.agecon.purdue.edu/resources/ download/1860.pdf (07/08/2012).

Tavares, E.C.N.; Carvalho, T.B. e De Zen, S. 2009. Rentabilidade econômica da bovinocultura de corte no estado de Goiás. Congresso da Sociedade Brasileira de Economia, Administração e Sociologia Rural, 47, 2006. Anais... Porto Alegre.

Zimmer, A.H. e Euclides Filho, K. 1997. As pastagens e a pecuária de corte brasileira. Simpósio internacional sobre Produção Animal em Pastejo. Anais... UFV. Viçosa. pp. 349-379.

Archivos de zootecnia vol. 63, núm. 241, p. 198. 